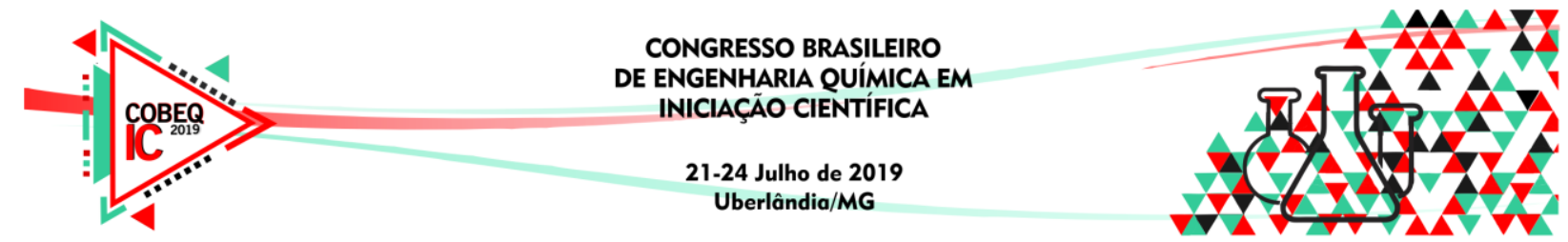

\title{
IMPLEMENTAÇÃO DE INTERPOLADORES MULTILINEARES COMO MODELOS SIMPLIFICADOS DE REATORES DE TRANSESTERIFICAÇÃO ETANÓLICA NO SIMULADOR GLOBAL EMSO
}

\author{
Christian O. Martins ${ }^{1}$, Felipe F. Furlan ${ }^{1}$, Roberto C. Giordano ${ }^{1}$ \\ ${ }^{1}$ Universidade Federal de São Carlos, Departamento de Engenharia Química \\ E-mail para contato: christiancb7@hotmail.com
}

\begin{abstract}
RESUMO - A análise tecno-econômica de processos químicos normalmente é realizada a partir de simulações estacionárias. Quando processos intrinsicamente dinâmicos estão presentes, meta-modelos normalmente são utilizados, evitando a resolução dinâmica de todo o sistema. Porém, alguns meta-modelos ocasionam grande perda de informação do modelo dinâmico por não aproximar corretamente as respostas principalmente com relação ao tempo, por seu comportamento nãolinear. Quando a acurácia do meta-modelo é essencial, grande número de parâmetros é necessário para capturar o comportamento dinâmico. No presente trabalho, uma alternativa foi proposta baseada em um meta-modelo híbrido que combina a resposta fornecida pela solução analítica de uma aproximação linear do modelo dinâmico acoplada a resposta de um meta-modelo do tipo interpolador multilinear. Essa abordagem foi aplicada ao reator de transesterificação homogênea básica do óleo de soja com etanol. Tal aproximação permitiu, por exemplo, a diminuição do número de pontos do interpolador para atingir a tolerância especificada na previsão de 645 para 195, para uma acurácia de 0,01 $\mathrm{mol} / \mathrm{L}$.
\end{abstract}

\section{INTRODUÇÃO}

Diante da necessidade moderna por fontes de energia sustentável, a procura por combustíveis renováveis vem crescendo. Desta forma, opções derivadas de uso de biomassa vêm chamando a atenção. Entre elas está o biodiesel, que é identificado como éster monoalquílico de um ácido graxo, tendo como matéria-prima fontes naturais e renováveis, como óleos vegetais e gordura animal (DIAS, 2016).

A produção do biodiesel ocorre por uma reação de transesterificação de um triacilglicerídeo com um álcool, podendo ser catalisado de forma homogênea por ácidos ou bases, ou heterogênea por óxidos de metais alcalinos terrosos, zeólitas, etc. Comumente é usado metanol ou etanol como composto alcoólico na reação, entretanto existe um viés pela produção etanólica no pais, dado o potencial da indústria de açúcar e álcool e a possibilidade de assim, obter um combustível completamente renovável (GUERRERO, 2011).

Nesse contexto, o trabalho está inserido no temático da FAPESP 2014/21252-0, que tem como objetivo estudar processos de produção de bioprodutos e biocombustíveis originados de 


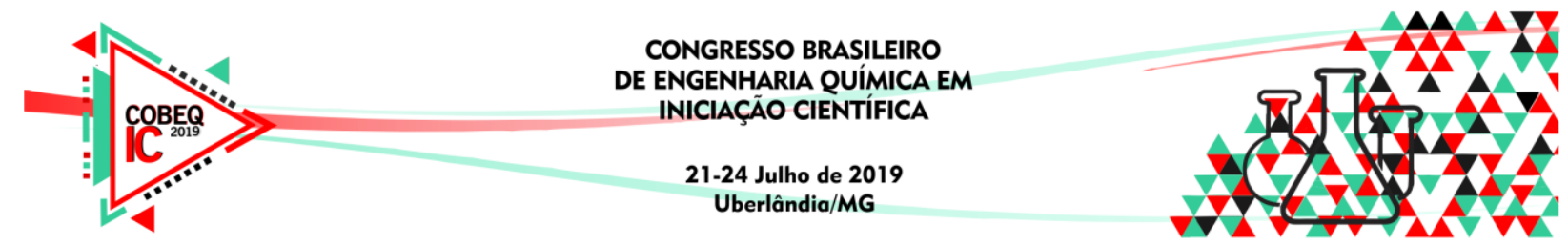

biomassas ricas em óleos e carboidratos. Uma das vertentes desse temático é a simulação de uma planta global de uma biorrefinaria que objetiva produzir tais produtos, com foco em biocombustíveis como etanol de primeira e segunda geração e biodiesel. O trabalho então possui como meta simular o processo de produção do biodiesel em um reator homogêneo em batelada através do software de simulação EMSO (Environment for Modeling Simulation and Optimization) (SOARES, 2003). O EMSO é um simulador de processos. Estes programas são essenciais para análise e otimização de processos envolvendo tecnologias, sejam novas, já implementadas, ou em desenvolvimento, tanto em questão de viabilidade econômica, quanto técnica. Isso é possível graças a possibilidade de descrever os processos através de modelos matemáticos, o que leva a um sistema de equações que pode conter de unidades a milhares de variáveis, a qual quando resolvida permite a melhor compreensão dos processos industriais em questão.

De modo geral, a análise e otimização técnica, econômica e ambiental de processos é realizada a partir de simulações estacionárias. Isso faz com que processos essencialmente transientes, como é o caso de reações em batelada ou batelada alimentada, sejam representados a partir de modelos simplificados (muitas vezes reatores estequiométricos). Com isso, perde-se muita informação sobre a influência das condições de operação do processo na resposta deste, por exemplo, tempo de reação, concentrações iniciais dos reagentes, temperatura, etc. Nesse contexto, o uso de meta-modelos se apresenta como alternativa interessante, por apresentar uma resposta aproximada da resposta do modelo rigoroso composto de equações diferenciais cuja solução analítica não é possível.

\section{METODOLOGIA}

A reação de produção de biodiesel por rota homogênea pode ser representada pelo seguinte modelo, descrito por Dias (2016), que utiliza como catalisador etóxido de sódio e descreve a reação a partir de pseudocomponentes que representam os triacilglicerídeos de um óleo (TG), os diacilglicerídeos (DG), os monoacilglicerídeos (MG), os ésteres etílicos que compõem o biodiesel (EE), além do glicerol (GL) e do etanol (ET). As cinéticas químicas das reações que compões esse modelo podem ser representadas pelas equações abaixo:

$$
\begin{aligned}
& r_{T G}=-k_{1} \times C_{T G} \times C_{E T}+k_{2} \times C_{D G} \times C_{E E} \\
& r_{D G}=k_{1} \times C_{T G} \times C_{E T}-k_{2} \times C_{D G} \times C_{E E}-k_{3} \times C_{D G} \times C_{E T}+k_{4} \times C_{M G} \times C_{E E} \\
& r_{M G}=k_{3} \times C_{D G} \times C_{E T}-k_{4} \times C_{M G} \times C_{E E}-k_{5} \times C_{M G} \times C_{E T}+k_{6} \times C_{G L} \times C_{E E} \\
& r_{E E}=k_{1} \times C_{T G} \times C_{E T}-k_{2} \times C_{D G} \times C_{E E}+k_{3} \times C_{D G} \times C_{E T}-k_{4} \times C_{M G} \times C_{E E} \\
& +k_{5} \times C_{M G} \times C_{E T}-k_{6} \times C_{G L} \times C_{E E} \\
& r_{G L}=k_{5} \times C_{M G} \times C_{E T}-k_{6} \times C_{G L} \cdot C_{E E} \\
& r_{E T}=-r_{E E}
\end{aligned}
$$

Onde " $r_{i}$ " é a velocidade global de reação de cada componente "i" (TG, DG, MG, EE, GL e ET) e " $\mathrm{C}_{\mathrm{i}}$ " a concentração de cada componente. 


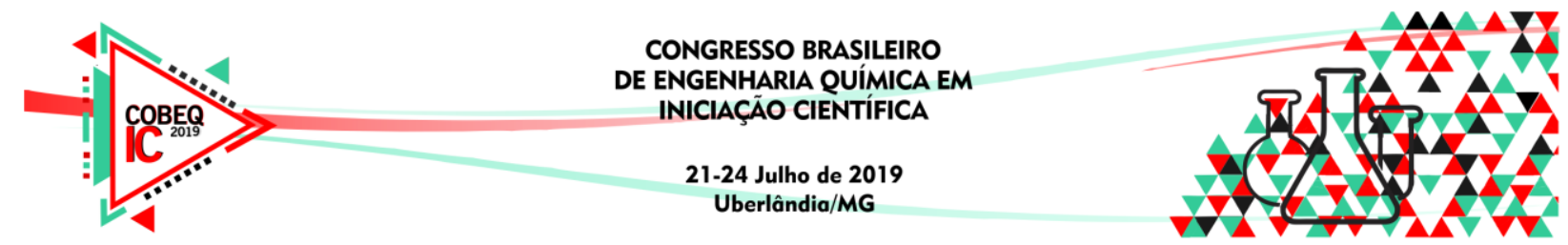

Para aplicação do trabalho, dentre as opções de óleos e temperaturas, cujos parâmetros cinéticos já haviam sido estimados na bibliografia, foi escolhido trabalhar com o óleo de soja e a temperatura de 308,15 K. Maiores informações sobre o modelo podem ser encontradas em Dias (2016).

A abordagem ao tema tomou duas frentes: produzir um interpolador puro, que trabalha diretamente como uma malha contendo os valores de resposta para uma série de pontos; e a outra de produzir um interpolador que complementa um modelo simplificado de equações exponenciais que representam uma aproximação rústica do modelo rigoroso. Essa segunda opção surge como alternativa para reduzir a quantidade de pontos necessários para produzir um interpolador com a precisão desejada. Com este fim, as equações de (1) a (6) foram modificadas de modo que formassem um sistema de equações diferenciais com solução algébrica conhecida que ainda se aproximassem do modelo rigoroso, representando o caráter dinâmico do sistema, cabendo ao interpolador corrigir apenas o desvio entre o modelo rigoroso e este modelo simplificado, que ainda apresenta um comportamento não linear, mas com uma menor variação.

As considerações feitas para possibilitar o modelo simplificado foram: a concentração do etanol é considerada constante durante a reação; as cinéticas das reações reversas são desprezadas; A solução para a cinética de reação para formação e consumo dos acilglicerois é definida analiticamente, dos outros componentes é definida pela estequiometria. Tais condições geraram um sistema de equações diferenciais ordinárias cuja solução resultou no seguinte sistema:

$$
\begin{aligned}
& C_{T G}=C_{T G_{0}} \cdot e^{-k_{1}^{t} \cdot t} \\
& C_{D G}=\frac{k_{1}^{J} \cdot C_{T G_{0}}}{k_{\mathrm{a}}^{J}-k_{1}^{J}} \times\left(e^{-k_{1}^{J} \cdot t}-e^{-k_{\mathrm{a}}^{J}-t}\right)+C_{D G_{0}} \times e^{-k_{\mathrm{a}}^{J} \cdot t}
\end{aligned}
$$

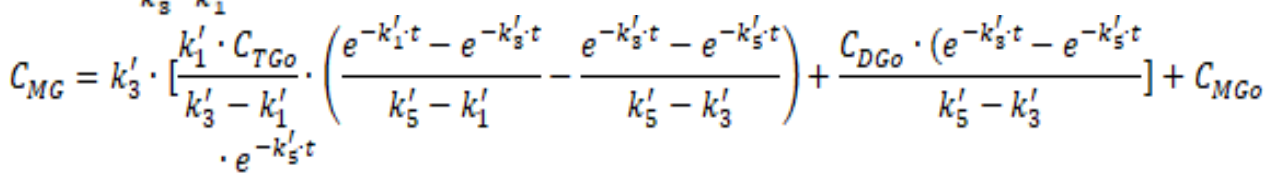

Onde os coeficientes ' $\mathrm{k}$ ' $_{\mathrm{i}}$ representam pseudoconstantes cinéticas formados pelo produto da concentração de etanol e " $\mathrm{k}_{\mathrm{i}}$ ", que é o parâmetro da constante cinética, sendo reajustado para o novo modelo.

Como o sistema só apresenta três reações independentes, só se fez necessário obter as concentrações dos acilgliceróis a partir da cinética. As demais concentrações foram obtidas a partir da estequiometria e aplicando balanços de massa.

Foi definido que as variáveis de entrada para o interpolador seriam o tempo e a proporção molar inicial entre etanol e óleo de soja, sendo a concentração inicial de óleo de soja utilizada de $0,7 \mathrm{~mol} / \mathrm{l}$, o mesmo valor utilizado durante os experimentos da referência utilizada (DIAS, 2016).

No caso do meta-modelo híbrido, o interpolador foi responsável por ajustar o desvio entre a resposta do modelo rigoroso e do modelo simplificado, como mostrado na equação 10. Assim, a resposta do meta-modelo híbrido é composta pela soma da resposta do modelo 


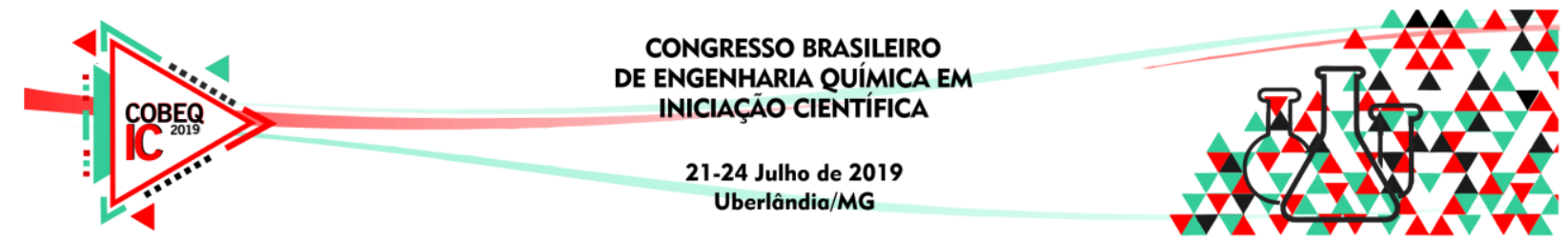

simplificado e do interpolador (Equação 11). Para a construção da malha, utilizou-se um programa construído em Matlab® descrito por Lino (2018) que realiza a construção de malhas refinando-as até que elas até que o erro máximo absoluto (MAE, Equação 12) atinja uma tolerância especificada.

$$
\begin{aligned}
& \text { desvio }_{\text {acilglicerídeo }}=C_{\text {acilglicerídeo }}^{\text {modelo rigoroso }}-C_{\text {acilglicerídeo }}^{\text {modelo simplifado }} \\
& C_{\text {acilglicerídeo }}^{\text {meta-modelo }}=C_{\text {acilglicerídeo }}^{\text {modelo simplifica }}+\text { desvio } \\
& M A E=\max \left(\left|C_{\text {acilgliglicerídeo }}^{\text {metádeo }}-C_{\text {acilglicerídeo }}^{\text {modelo }}\right|\right)
\end{aligned}
$$

\section{RESULTADOS}

Inicialmente foi realizado o ajuste das pseudoconstantes do modelo simplificado. Os dados utilizados foram gerados a partir da simulação do modelo rigoroso retornando valores de concentração de TG, DG e MG variando o tempo de 0,5 em 0,5 minutos até 25 minutos e a proporção molar de etanol/óleo de soja variando de 6 a 12 com espaçamento entre os pontos de 0,5 . Obteve-se os valores de $\mathrm{k}_{\mathrm{TG}}$ de $0,0777 \mathrm{~L} \cdot \mathrm{mol}^{-1} \cdot \mathrm{min}^{-1}, \mathrm{k}_{\mathrm{DG}}$ de $0,0523 \mathrm{~L} \cdot \mathrm{mol}^{-1} \cdot \mathrm{min}^{-1} \mathrm{e}$ $\mathrm{k}_{\mathrm{MG}}$ de $0,0522 \mathrm{~L} \cdot \mathrm{mol}^{-1} \cdot \mathrm{min}^{-1} \mathrm{e}$ os coeficientes de determinação linear resultantes foram 0,99 , 0,83 e 0,85 , respectivamente.

O desvio entre o comportamento do modelo simplificado e o modelo rigoroso e as concentrações calculadas pelo modelo simplificado podem ser visualizados na Figura 2 por um tempo de reação de 25 min com proporção inicial de etanol/óleo de soja de 9/1, para os mono (MG), di (DG) e triacilglicerídeos (TG).

Figura 2 - À esquerda o desvio entre as concentrações dadas pelos dois modelos e concentração calculada pelo modelo simplificado. À direita o perfil cinético das reações representado pelo modelo rigoroso (índice "r") e pelo modelo simplificado (índice "s").

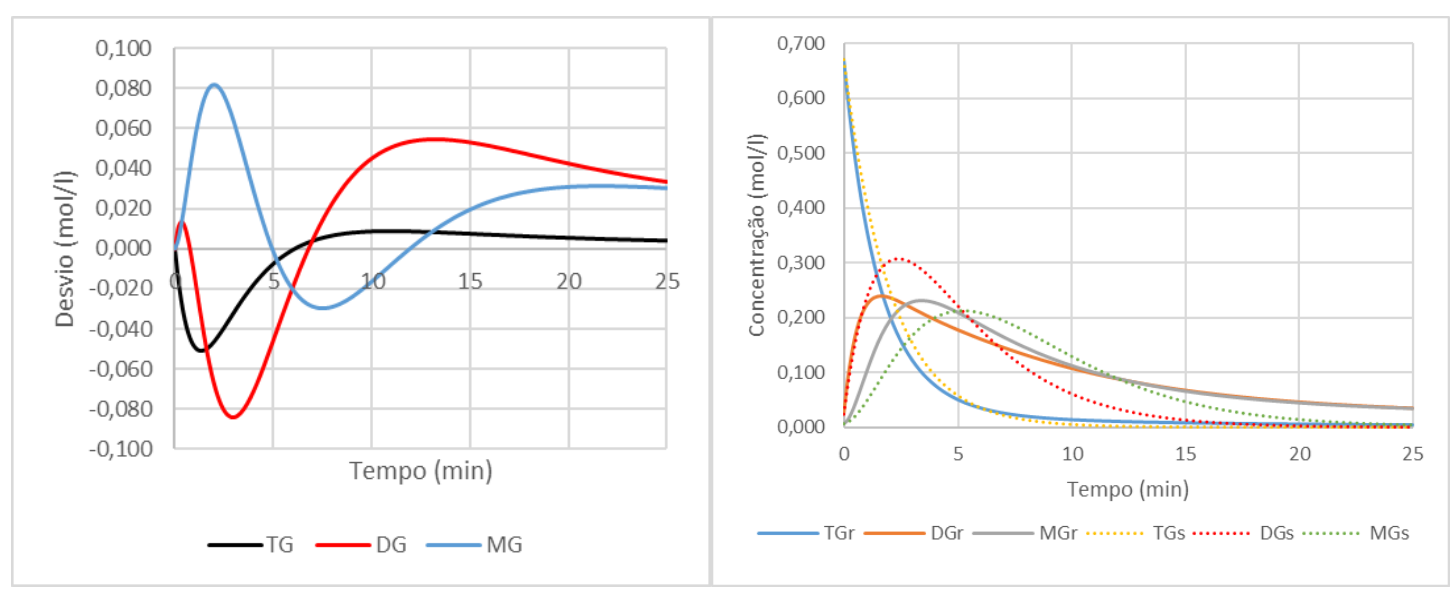

Para a construção do meta-modelo híbrido, consistindo do modelo simplificado e do interpolador multilinear, considerou-se um erro máximo absoluto. A malha utilizada pelo interpolador foi escolhida como aquela que atingia o erro máximo especificado, a partir de um teste de 1000 pontos no intervalo de validade para as variáveis tempo de reação e proporção 

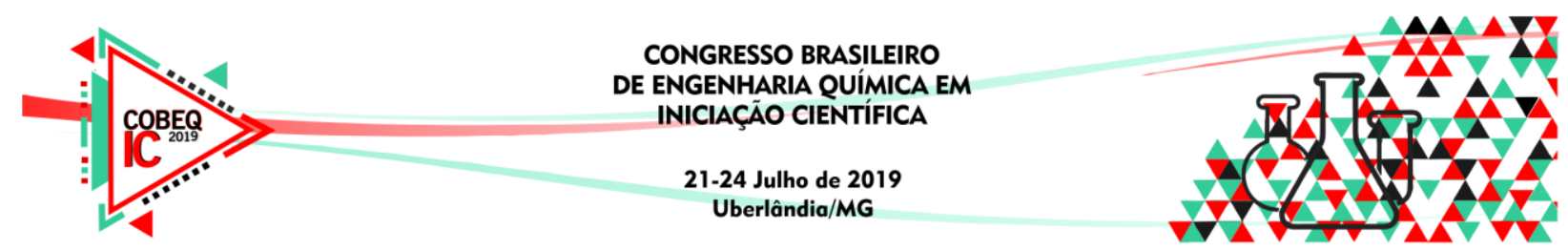

etanol/óleo. Podemos analisar, na Figura 3 que a aproximação do meta-modelo híbrido ao modelo rigoroso melhora a medida que o limite para o erro máximo absoluto diminui, como esperado. Observa-se que com tolerância de 0,01 mol/l, o modelo híbrido já representa de forma praticamente exata a resposta do modelo rigoroso.

Figura 3 - Gráficos da concentração dos meta-modelo pela concentração do modelo rigoroso para o DG com proporção etnaol/óleo de soja inicial de 9/1. "Cs" é o valor da concentração a partir do modelo simplificado sozinho, " $\mathrm{Cx}$ " do meta-modelo, onde " $\mathrm{x}$ " (em mol/l) é a tolerância determinada para a escolha do tamanho da malha e "Cr" do modelo rigoroso.

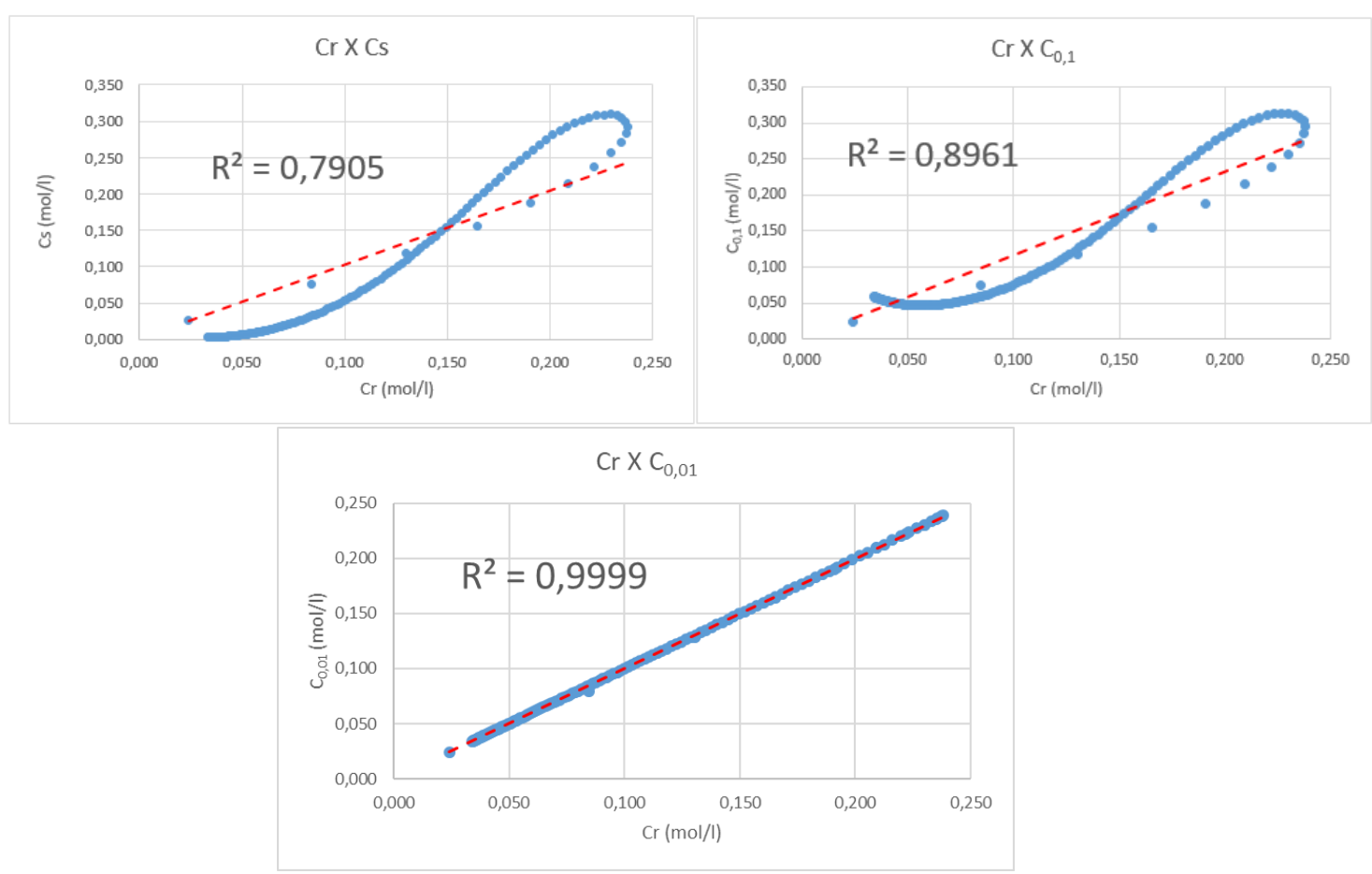

Finalmente, o modelo híbrido foi comparado ao meta-modelo composto somente pelo interpolador multilinear. Para isso, comparou-se o número de pontos necessários da malha para o meta-modelo atingir determinada precisão. Observa-se na Figura 4 que o modelo híbrido, contendo a solução analítica das equações diferenciais simplificadas e o interpolador precisou de um número expressivamente menor de pontos, a medida que a precisão requerida aumentou (195 pontos vs. 645 pontos, para a precisão de 0,01), o tempo de simulação para o modelo rigoroso foi de $0,239 \pm 0,009 \mathrm{~s}$.

Tabela 1 -Tabela da tolerância, do número de pontos necessários para o meta-modelo híbrido e com o interpolador puro e do tempo de simulação.

\begin{tabular}{|c|c|c|c|c|}
\hline $\begin{array}{c}\text { Tolerância } \\
\text { (mol/l) }\end{array}$ & $\begin{array}{c}\text { Pontos do } \\
\text { meta-modelo } \\
\text { hibrido }\end{array}$ & $\begin{array}{c}\text { Pontos do } \\
\text { interpolador } \\
\text { puro }\end{array}$ & $\begin{array}{c}\text { Tempo de } \\
\text { simulação para o } \\
\text { modelo hibrido (s) }\end{array}$ & $\begin{array}{c}\text { Tempo de simulação } \\
\text { para o interpolador } \\
\text { puro (s) }\end{array}$ \\
\hline 0,01 & 195 & 645 & $0,121 \pm 0,009$ & $0,35 \pm 0,02$ \\
\hline 0,1 & 4 & 34 & $0,016 \pm 0,003$ & $0,046 \pm 0,009$ \\
\hline
\end{tabular}




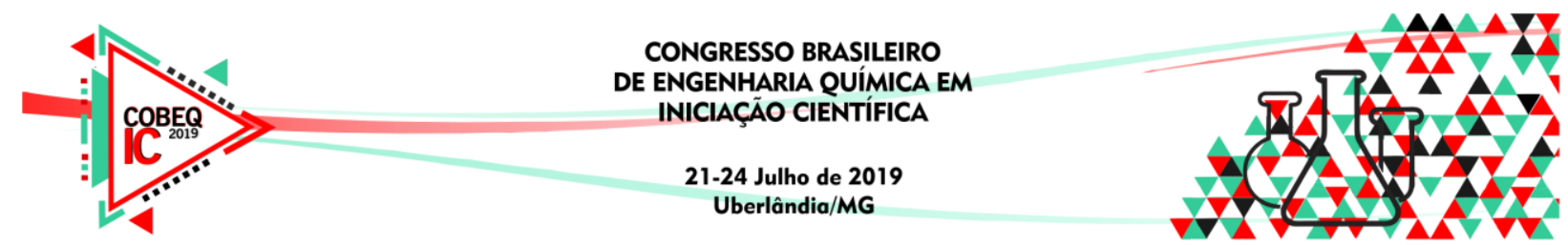

\section{CONCLUSÕES}

A utilização de meta-modelos do tipo tabela de inspeção multilinear para a aproximação de modelos dinâmicos em simulações estacionárias leva a números elevados de parâmetros, devido ao comportamento não-linear dos modelos dinâmicos, principalmente com relação ao tempo. O meta-modelo híbrido proposto permitiu a diminuição da complexidade do modelo ao utilizar uma aproximação linear das equações diferenciais, cuja solução analítica é possível. Com isso, o número de pontos necessários para que o metamodelo atingisse determinada acurácia diminuiu entre 70 e $88 \%$, dependendo da acurácia escolhida. O meta-modelo foi utilizado no estudo de caso da reação de transesterificação básica homogênea do óleo de soja. Porém, a abordagem é geral e pode ser empregada a qualquer sistema de equações diferenciais.

\section{REFERÊNCIAS}

DIAS, T.P.V.B., ESTUDO DO EQUILÍBRIO LÍQUIDO-LÍQUIDO E CINÉTICA DE REAÇÃO NA PRODUÇÃO DE ÉSTERES ETÍlLICOS. 2016. 171f. Tese de Doutorado UNIVERSIDADE ESTADUAL DE CAMPINAS, Campinas-SP, 2016.

FURLAN F.F., COSTA C.B.B., FONSECA G.C., SOARES R.P., SECCHI A.R., CRUZ A.J.G., GIORDANO R.C. Assessing the production of first and second generation bioethanol from sugarcane through the integration of global optimization and process detailed modeling. Computers and Chemical Engineering 43:1-9, 2012.

FURLAN F.F.; TONON F.R.; PINTO F.H.P.B.; COSTA C.B.B.; CRUZ A.J.G.; GIORDANO R.L.C.; GIORDANO R.C. Bioelectricity versus bioethanol from sugarcane bagasse: is it worth being flexible? Biotechnology for Biofuels 6:142, 2014.

FURLAN F.F.; LINO A.R.A.; MATUGI K.; CRUZ A.J.G.; GIORDANO R.C. A simple approach to improve the robustness of equation-oriented simulators: Multilinear look-up table interpolators. Computers and Chemical Engineering 86:1-4, 2016.

GUERRERO, J.K.R. SÍNTESE DE BIODIESEL A PARTIR DE ÓLEO DE MAMONA EMPREGANDO ETANOL SUBCRÍTICO. 2011. 126f. Dissertação do MestradoUniversidade Estadual de Campinas, Campinas, 2011.

LINO, A. R. A., Interpoladores multilineares como metamodelos: Integração à simulação de biorrefinarias no ambiente EMSO. 2018. 131f. Dissertação de Mestrado - UNIVERSIDADE FEDERAL DE SÃO CARLOS, São Carlos-SP,2018.

SOARES, R.P.; SECCHI, A.R. EMSO: A new environment for modelling, simulation and optimization. Computer Aided Chemical Engineering, v. 14, pg 947-952, 2003. 